\title{
Evaluation of the Michigan Disease Surveillance System for Histoplasmosis Reporting
}

\author{
Veronica A. Fialkowski, Leigh M. Tyndall Snow, Kimerbly Signs and Mary Grace \\ Stobierski
}

Emerging and Zoonotic Infectious Diseases Section, Michigan Department of Health and Human Services, Lansing, MI, USA

\section{Introduction}

Histoplasmosis is an infectious disease caused by a fungus called Histoplasma capsulatum. Fungal spores are found in the soil, mostly associated with bird and bat droppings, and if inhaled can cause lung infection. Histoplasmosis is a reportable disease in Michigan and a case definition was implemented in 2007. Cases are reported into the Michigan Disease Surveillance System (MDSS), a web-based electronic database, and investigated by local health departments (LHD). An evaluation of the histoplasmosis surveillance system was conducted.

\section{Methods}

The histoplasmosis surveillance system was evaluated using the 2001 Centers for Disease Control and Prevention Updated Guidelines for Evaluating Public Health Surveillance Systems. Acceptability was assessed by matching a comprehensive list of hospitals in Michigan to hospitals that directly report into MDSS. To evaluate timeliness, average times between diagnosis date, date reported to LHD, and investigation completion date were calculated. Completed cases from 2014 were reviewed to determine if cases met the case definition criteria. Positive predictive value (PPV) was then calculated for cases classified as a case in MDSS, but that did not meet the case definition upon review.

\section{Results}

From 2004 to 2014, a total of 1,608 confirmed or probable cases were reported into MDSS, with a slight increasing trend in case numbers over time. Overall, MDSS is simple to use and fairly flexible, allowing for changes and adaptations to the case report form. Overtime the proportion of cases classified as not a case to cases classified as confirmed or probable has increased. In 2014, $72 \%$ of histoplasmosis cases reported into MDSS did not meet the case definition. Between 50 and $70 \%$ of hospitals in each region of Michigan use MDSS to report, which shows a reasonable acceptance of the Michigan reporting system. Cases were reported to MDSS or a LHD a mean of 14 days after diagnosis $(n=729)$. On average, case investigations took 35 days to complete $(n=1,145)$. Prior to 2007 , case investigation time averaged 48 days, and decreased to a mean of 31 days after the implementation of the case definition in 2007. In 2014, 124 cases were reported as confirmed or probable, and were marked completed at the end of the respective year. After the state's review of cases, 50 cases (40 percent) were classified incorrectly and needed follow-up. The PPV was 79.84\% (95\% CI: 71.7\%-86.5\%).

\section{Conclusions}

Michigan's histoplasmosis surveillance system is relatively simple, but the misclassification of cases is troublesome. Development of tools for LHDs to aid in classification of cases may improve the PPV and decrease case investigation time. Increasing the number of hospitals that report directly to MDSS would indicate more acceptability, and increase sensitivity. There are advantages of increased use of electronic laboratory and the shift toward electronic medical records, such as an increase in number of cases reported to public health, however, the number of cases classified as 'not a case' increases as well, which my increase LHD workload.

\section{Keywords}

surveillance; evaluation; fungal disease

\section{Acknowledgments}

This study/report was supported in part by an appointment to the Applied Epidemiology Fellowship Program administered by the Council of State and Territorial Epidemiologists (CSTE) and funded by the Centers for Disease Control and Prevention (CDC) Cooperative Agreement Number 1U38OT000143-02

MDHHS Bureau of Disease Control, Prevention, \& Epidemiology Staff: Edward Hartwick, MS, Tiffany Henderson, MPH

\section{References}

1. German RR, Lee LM, Horan JM, et al. Updated guidelines for evaluating public health surveillance systems: recommendations from the Guidelines Working Group. MMWR Recomm. Rep. 2001; 50 (RR13):1-35. 2. Lenhart SW, Schafer MP, Singal M, et al. Histoplasmosis: Protecting Workers at Risk. DHHS (NIOSH). 2004; 2005-109: 1-26 3. Wheat LJ, Freifeld AG, Kleiman MB, et al. Clinical Practice Guidelines for the Management of Patients with Histoplasmosis: 2007 Update by the Infectious Diseases Society of America. Clin. Infect. Dis. 2007; 45: 807-25. 4. Whitfield K, Kelly H. Using the two-source capture-recapture method to estimate the incidence of acute flaccid paralysis in Victoria, Australia. World Health Organization. 2002; 80 : 846-851.

\footnotetext{
*Veronica A. Fialkowski

E-mail: FialkowskiV@michigan.gov
} 